\title{
Pressure Loss Reduction in U-Bend Using the Adjoint Method
}

\author{
Ahmed Osama $^{*}$, Khairy Elsayed ${ }^{\dagger}$, Mohamed M. El-Telbany ${ }^{\ddagger}$
}

\begin{abstract}
Gas turbine blades are usually cooled by air, which flows through internal passages serpentine shaped, coming from a high pressure compressor to save it from the high temperature. Using 180 degree turns to connect internal paths led to high pressure loss in the cooling system. The purpose of this study is reducing the pressure loss using the adjoint solver method to get the optimum shape of U-shaped paths with and without guide vane $(\mathrm{GV})$. Using three different cases with three different regions of modifications. The optimized shapes showed a reduction in the pressure loss up to $60 \%$ in the case of U-bend without guide vanes.
\end{abstract}

Keywords: U-Bend, Pressure loss, Adjoint method, Shape optimization.

\section{Introduction}

In gas turbines, increasing inlet gas temperature will increase the output power, however there is a restriction from blades material. Blade cooling is essential to keep it safe from high temperature, so bled air is extracted to cool the blades by pathing through internal passages (180-degrees turn). These turns have an aggressive significant effect on the pressure loss due to the phenomena of separation which occurs downstream the 180 degree turn, made it responsible for high loss of the entire cooling system loss, and have a reverse effect on heat transfer.

The literature has many investigations on the U-turn geometry. Sudo et al. [1] experimentally investigated the turbulent flow through a circular sectioned $180^{\circ}$ bend founding the effect of different physical quantities in different regions. Kim and Lee [2] investigated the structure of 3D flow within a curved micro tube for varying Dean numbers using micro digital holographic particle tracking velocimetry. Their results showed that for low Dean number, the secondary flow did not develop. Miloude et al. [3] conducted a computational study on turbulent flow in a circular U-bend to select the most accurate turbulence model. They stated that there were no significant differences between the two turbulence models $(\mathrm{k}-\varepsilon$ and the RSM models). Al-Yaari and Abu-Sharkh [4] studied the effect of the inlet velocity and bend to pipe radius ratio on oil-water phase separation in $180^{\circ}$ bend. Their results showed that as the inlet velocity or bend to pipe ratio increases, the separation in bend region decreases.

Demonstrator, Mechanical Power Engineering Department, Faculty of Engineering-Mataria, Helwan University, Masaken El-Helmia P.O., Cairo 11718, Egypt., Email: ahmedelshaier92@ gmail.com

$\uparrow$ Assistant professor, Mechanical Power Engineering Department, Faculty of Engineering- Mataria, Helwan University, Masaken El-Helmia P.O., Cairo 11718, Egypt., Email: kelsayed75@ gmail.com Professor/ Senior research scientist, Vrije Universiteit Brussel, Department of Mechanical Engineering, Research Group Fluid Mechanics and Thermodynamics, Pleinlaan 2, 1050 Brussels, Belgium.

* Professor, Mechanical Power Engineering Department, Faculty of Engineering- Mataria, Helwan University, Masaken El-Helmia P.O., Cairo 11718, Egypt., Email: profeltelbany@yahoo.com 
Sewall and Taffi [5] used large eddy simulation to study the flow and heat transfer in a $180^{\circ}$ bend to investigate the effect of inserting a rib in the bend region. They stated that the rib would increase pressure drop and heat transfer. Luo and Razinsky [6] investigated the flow in $180^{\circ}$ turning ducts with and without guide vanes. Schüler et al. [7] studied the effect of three different geometries of turning vanes, inner, outer, and both, located in the bend region on pressure loss and heat transfer of a ribbed rectangular two-pass internal cooling channel. They stated that the inner vane had the lowest pressure loss while outer vane had the worst. Saha and Acharya [8] studied the effect of nine bend geometries on the heat transfer and the pressure drop in a two-pass coolant square channel. Their results showed that using turning vanes is recommended for reducing pressure loss at the cost of heat transfer. Lee et al. [9] also studied the effect of turning vanes in the tip turn region of rectangular duct on pressure loss and heat transfer. Following the same approach Xie et al. [10] studied the influences of guide ribs/vanes on enhanced heat transfer of a turbine blade tip-wall.

Verstraete and $\mathrm{Li}$ [11] followed another approach to reach the goal, getting optimum design for U-bend, using geometry parameterization using several 3D simulations. Their results showed that U-bend shape could change to minimize the pressure drop (69.2\% of original) or maximize the heat transfer rate (116.93\% of original). Elsayed et al. [12] followed the same approach but using the adjoint method on 2-D geometry reaching a pressure drop equals $68 \%$ of the original shape.

Based on the above mentioned studies, the flow in a U-bend still needs more investigations to obtain the optimum geometry. In the current study, the adjoint method will be used for different U-bend configurations (with and without guide vanes) to minimize the pressure drop.

\section{Numerical Settings}

\section{Geometry and Grid}

The baseline geometry is identical to that used by Saha et al. [8] as is shown in Fig. 1. The Ubend has a rounded corner with a square cross section area, Height $(\mathrm{H})=$ Width $(\mathrm{W})=$ $0.03048 \mathrm{~m}$, and the inlet and outlet paths have a length equal ten times the width downstream and upstream the turn region to ensure that the flow would get fully developed. The space between the inner walls of inlet and outlet equals $0.2 \mathrm{~W}$. The radius of the outer and inner turns in bend region keep the space equal to W. For guide vane 1 and guide vane 2 cases the vane has a thickness equals $0.05 \mathrm{~W}$ and located at the middle of bend region.

ANSYS meshing is used to generate the grid. Using multi zone (Hexa / Prism) method and inflation, to get layers close to the wall where the thickness of the first cell equals $4.4 \times 10^{-4} \mathrm{~m}$ with 1.2 growth rate. The minimum number of layers is three. The mesh independent study showed that reducing the cell size did not change the pressure drop between inlet and outlet significantly as shown in Fig. 2 and Table 1. Consequently, the medium mesh of each case will be used for subsequent analysis. 

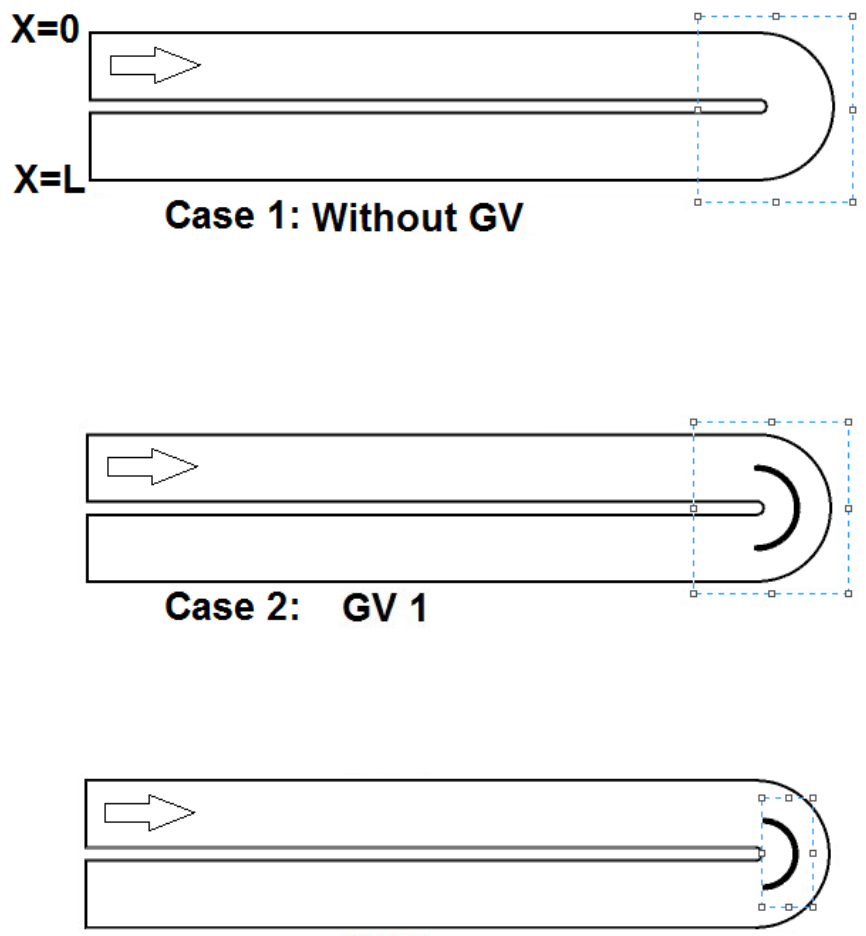

Case 3: GV 2

Fig. 1. Schematic diagrams for the three geometries. The main difference between GV-1 and GV-2 is the optimization domain.

Table 1: Mesh independent study results

\begin{tabular}{|c|c|c|c|}
\hline Case & Mesh size & $\Delta p(\mathrm{~Pa})$ & $\begin{array}{c}\text { Percentage } \\
\text { absolute difference }\end{array}$ \\
\hline \multirow{3}{*}{$\begin{array}{r}\text { 1: Without guide } \\
\text { Vane }\end{array}$} & 139400 & 221 & 3.27 \\
\hline & 625600 & 214 & 1.05 \\
\hline & 765000 & 211 & \\
\hline \multirow{3}{*}{$\begin{array}{l}\text { 2: With guide vane } \\
\text { and wide region } \\
\text { to modify }\end{array}$} & 53752 & 127 & 4.01 \\
\hline & 299536 & 122 & 1.28 \\
\hline & 860098 & 124 & \\
\hline \multirow{3}{*}{ 3: With guide vane } & 65244 & 128 & 1.38 \\
\hline & 293912 & 127 & .061 \\
\hline & 885600 & 126 & \\
\hline to modify & & & \\
\hline
\end{tabular}




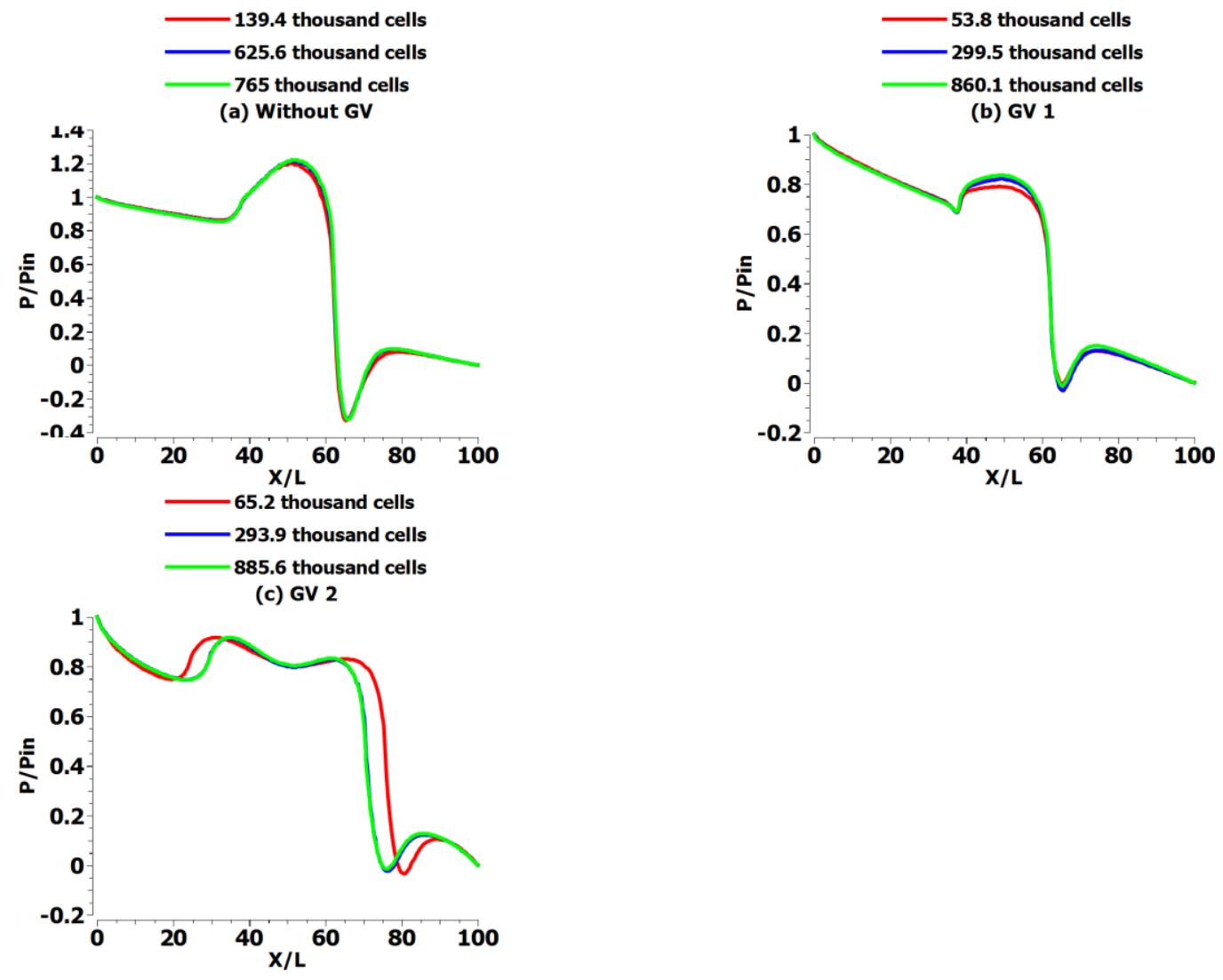

Fig. 2. Mesh independent study.

\section{Validation for the Baseline}

The friction factor can be estimated using Eq. (1) [8].

$$
f=\frac{\Delta p / \rho v^{2}}{4 \Delta x / D_{h}}
$$

where $\Delta \mathrm{x}$ is the length over which the friction factor is calculated. For fully developed flow in a smooth circular channel $f_{0}$ can be estimated using Eq. (2) [8].

$$
f_{0}=0.046 * R_{e}^{-0.2}
$$

Table 2: Validation for the original baseline shape

\begin{tabular}{|c|c|c|c|}
\hline Study & Saha et al. [8] & Present study & $\mid \%$ difference $\mid$ \\
\hline$f / f_{0}$ & 4.52 & 4.56 & 0.885 \\
\hline
\end{tabular}

\section{Computational Details}

The working fluid was air. The inlet velocity was $12 \mathrm{~m} / \mathrm{s}$ (Reynolds number $=25 \times 10^{3}$ ). Using standard k- $\varepsilon$ turbulence modeling with standard wall function. The pressure-velocity coupling was SIMPLE and the other spatial discretization settings were Green-Gauss cell based for gradient, standard for the pressure, second order upwind for the momentum, first order upwind for the turbulent kinetic energy, first order upwind for the turbulent dissipation rate. 


\section{The Adjoint Solver}

The adjoint method is an efficient method for shape optimization. It calculates the sensitivity of the shape change to the objective function. Using 20 points in each $\mathrm{x}$-direction and $\mathrm{y}$ direction, while 15 in z-direction. The boundary continuity had order of 2 . No symmetry in motion was allowed. Every case had a defined part which allowed to modify within restricted boundaries called bounding box. The bounding box is shown in Fig. 3 for without GV case, which is the same for guide vane case 1(the vane and the U-bend were allowed to modify together). While for guide vane case 2, the dimensions were $1.725 \mathrm{~W}$ in $\mathrm{x}$-direction, 0.9625 $\mathrm{W}$ in $\mathrm{y}$-direction, and $\mathrm{W}$ in $\mathrm{z}$-direction, so just the guide vane was allowed to modify and cannot exceed the U-bend body.

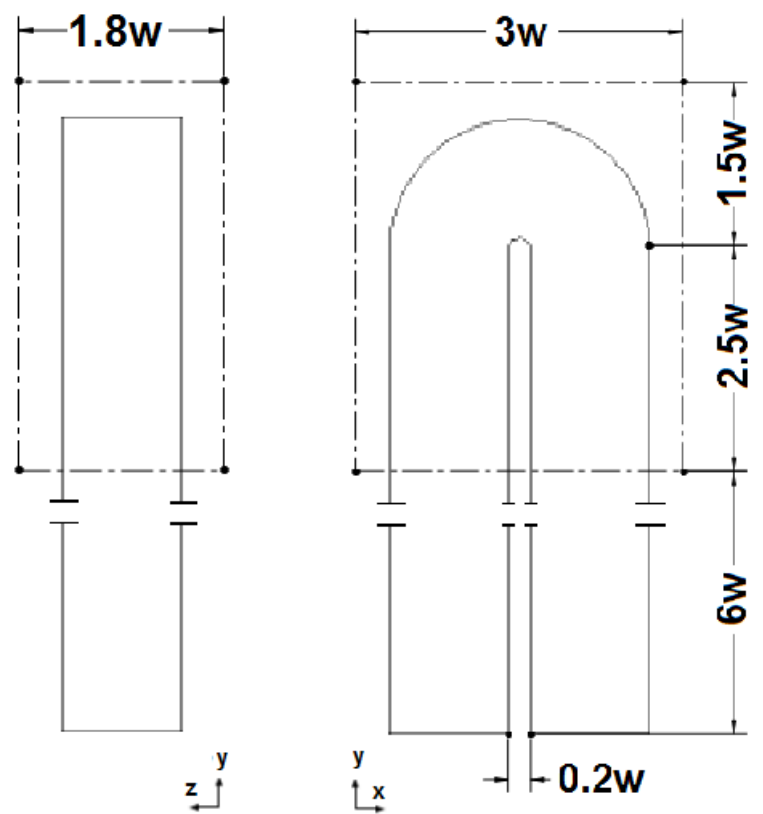

Fig. 3. The Bounding box (for the adjoint solver) for case 1 geometry.

\section{Adjoint Equations}

The cost function $\mathcal{J}$ (such as the pressure drop) is a function of the shape $\mathcal{F}$ and the flow field $\omega$, i.e.,

$$
\mathcal{J}=\mathcal{J}(\mathcal{F}, \boldsymbol{\omega})
$$

where the flow field $\boldsymbol{\omega}$ are related to $\mathcal{F}$ by the Navier-Stokes equations.

$$
\mathbf{R}(\mathcal{F}, \boldsymbol{\omega})=0
$$

If the shape is changed, the flow field will chang so that the variation of the cost function is

$$
\delta \mathcal{J}=\frac{\partial \mathcal{J}}{\partial \mathcal{F}} \delta \mathcal{F}+\frac{\partial \mathcal{J}}{\partial \omega} \delta \omega
$$


The $\delta \mathcal{F}$ term can be obtained directly from the geometry change, but the $\delta \boldsymbol{\omega}$ term will need flow field evaluations, which are time consuming. In order to eliminate the explicit dependence of $\delta \mathcal{J}$ on $\delta \boldsymbol{\omega}$, the following procedure is applied. Taking the variations of the flow governing equations, we have

$$
\delta \mathbf{R}=\frac{\partial \mathbf{R}}{\partial \mathcal{F}} \delta \mathcal{F}+\frac{\partial \mathbf{R}}{\partial \boldsymbol{\omega}} \delta \boldsymbol{\omega}=0
$$

Introducing an arbitrary multiplier $\boldsymbol{\psi}$, we may write

$$
\delta \mathcal{J}=\frac{\partial \mathcal{J}}{\partial \mathcal{F}} \delta \mathcal{F}+\frac{\partial \mathcal{J}}{\partial \boldsymbol{\omega}} \delta \boldsymbol{\omega}-\boldsymbol{\Psi}^{T}\left(\frac{\partial \mathbf{R}}{\partial \mathcal{F}} \delta \mathcal{F}+\frac{\partial \mathbf{R}}{\partial \boldsymbol{\omega}} \delta \boldsymbol{\omega}\right)
$$

or,

$$
\delta \mathcal{J}=\left(\frac{\partial \mathcal{J}}{\partial \boldsymbol{\omega}}-\boldsymbol{\Psi}^{T} \frac{\partial \mathbf{R}}{\partial \boldsymbol{\omega}}\right) \delta \boldsymbol{\omega}+\left(\frac{\partial \mathcal{J}}{\partial \mathcal{F}}-\boldsymbol{\Psi}^{T} \frac{\partial \mathbf{R}}{\partial \mathcal{F}}\right) \delta \mathcal{F}
$$

To eliminate the explicit dependence of $\delta \mathcal{J}$ on $\delta \boldsymbol{\omega}$, let

$$
\frac{\partial \mathcal{J}}{\partial \boldsymbol{\omega}}-\boldsymbol{\Psi}^{T} \frac{\partial \mathbf{R}}{\partial \boldsymbol{\omega}}=0
$$

This is the adjoint equation. The solution $\boldsymbol{\Psi}$ is called the co-state variable. The FLUENT adjoint solver uses the discrete adjoint approach to solve this equation for $\boldsymbol{\psi}$ and estimate the gradients.

Once $\boldsymbol{\Psi}$ is obtained, $\delta \mathcal{J}=\mathcal{G} \delta \mathcal{F}$ can be estimated, where $\mathcal{G}=\frac{\partial \mathcal{J}}{\partial \mathcal{F}}-\boldsymbol{\psi}^{T} \frac{\partial \mathbf{R}}{\partial \mathcal{F}}$ is the gradient of $\mathcal{J}$ with respect to the shape change $\delta \mathcal{F}$. Given the gradient, $\delta \mathcal{F}$ can be chosen to have a negative $\delta \mathcal{J}$ to reduce the cost function.

An improvement can be made by the shape change $\delta \mathcal{F}=-\lambda \mathcal{G}$ consequently, $\delta \mathcal{J}=-\lambda \mathcal{G}^{T}$ where $\lambda$ is the scale factor which control the geometry change as explained hereafter. After making such geometry modification, the gradient can be recalculated and the process repeated to follow the path of steepest descent until a minimum is reached. After several design cycles, when the cost function approaches zero change, the target design is accomplished. The optimization process using the adjoint solver is shown in Fig. 4.

\section{Results and Discussion}

For the case without $\mathrm{GV}$, the application of the adjoint method resulted in a reduction of $40.5 \%$ with respect to the original geometry. The final optimal shape is shown in Fig. 5. A comparison between the original and the optimal shape for case 1 is shown in Fig. 6 . The optimal pressure drop was 86.64 Pa. 


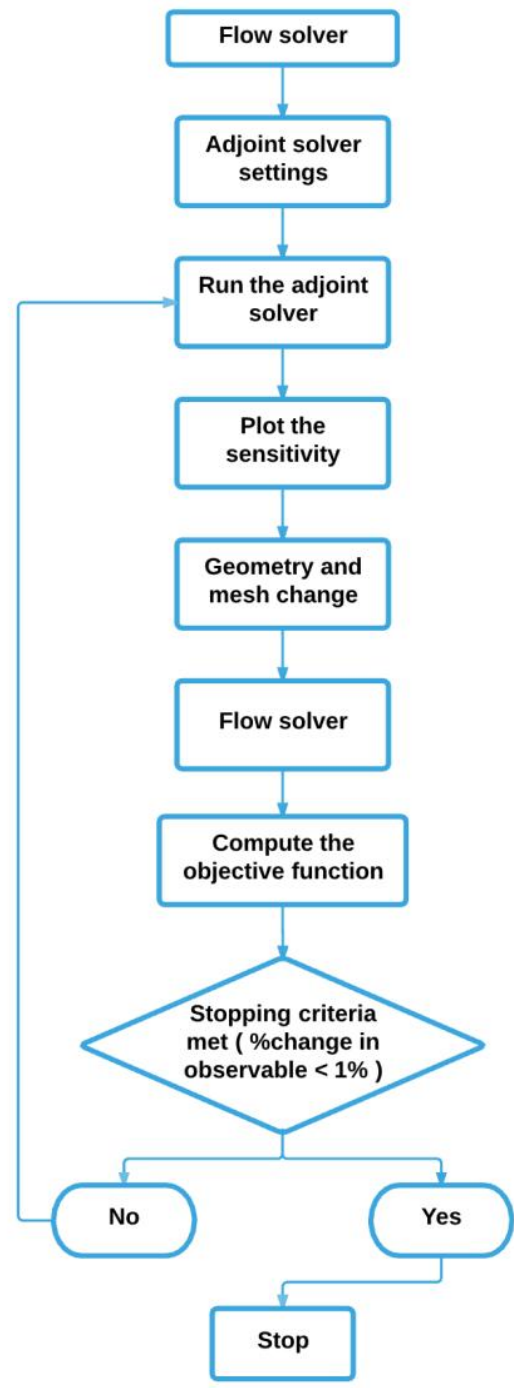

Fig. 4. The optimization process using the adjoint solver.
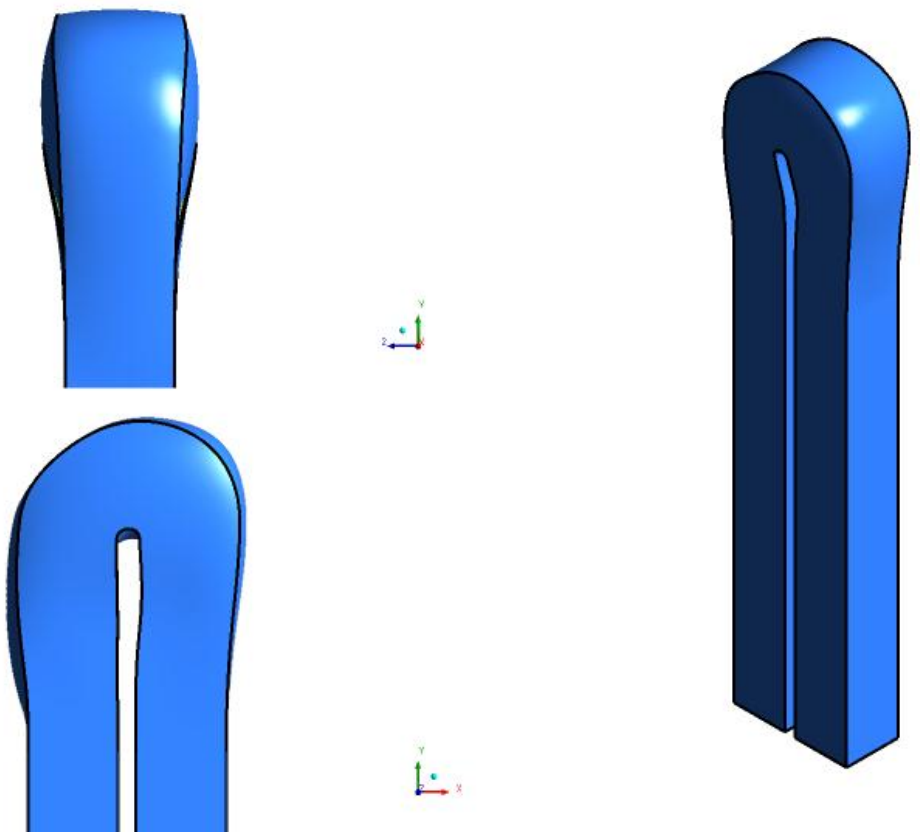

Fig. 5. Optimal shape for case 1. 


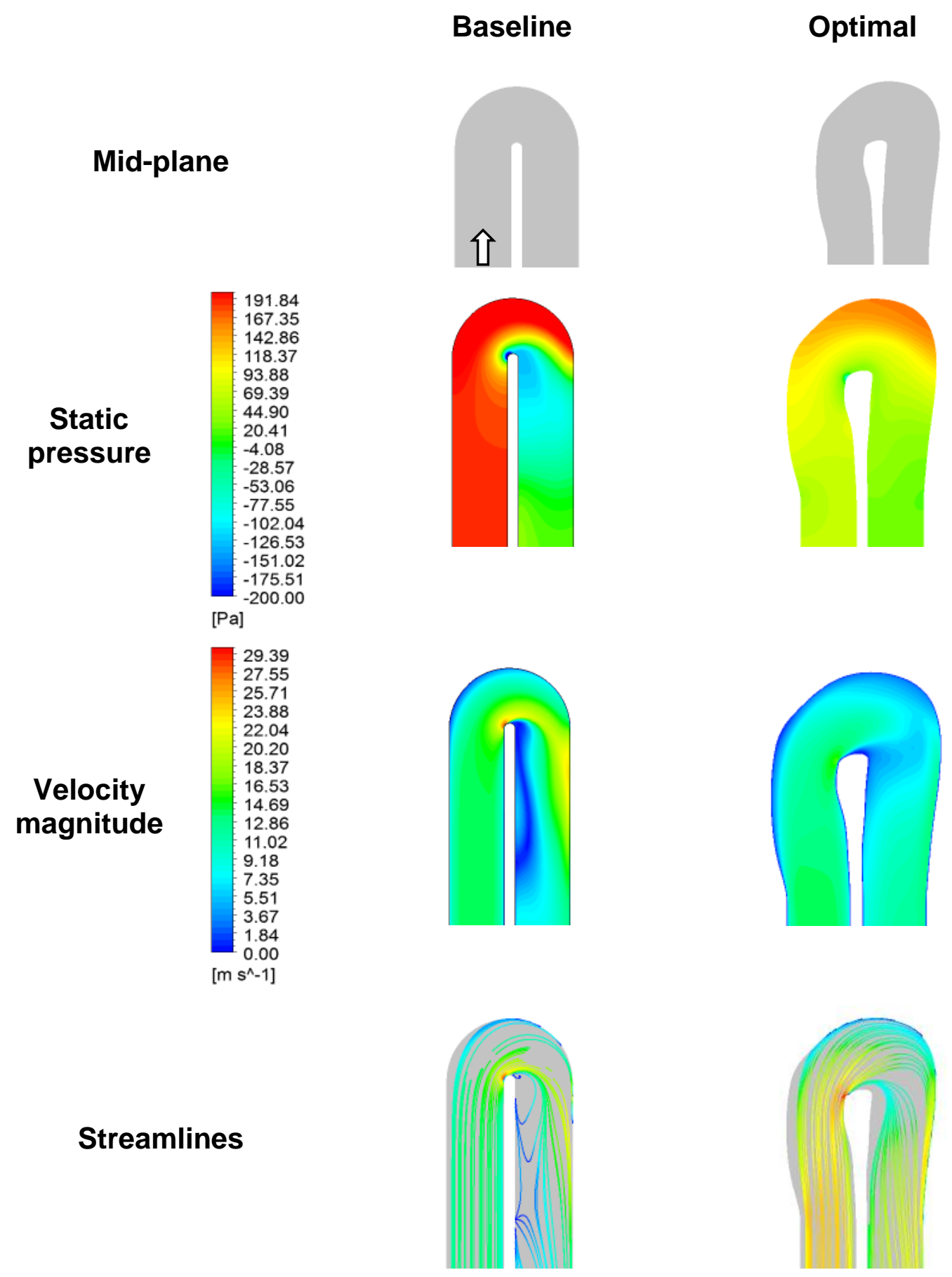

Fig. 6. Baseline and the optimal shape at the mid-plane. 
For the guide vane case 1, the pressure drop between the inlet and the outlet reduced to $67.1 \%$ of the original pressure drop which equals $82.14 \mathrm{~Pa}$. The optimal shape is shown in Fig. 7. The comparison between the original and the optimal shape is shown in Fig. 8.

The results for the guide vane case 2 are illustrated in Fig. 9. While the comparison is given in Fig. 10. The final pressure drop was $123.07 \mathrm{~Pa}$ which represents $97.2 \%$ of the original shape. The difference between the original shape and the optimum shape for all cases according to the pressure distribution divide by inlet pressure along the inner wall and outer wall at the mid-plane is shown in Fig. 11. The results show reductions in the separation regions which shown is clearly through the first case in Fig. 12. Fig. 13. presented the optimal shapes of the three tested configurations. While Table 3 shows the normalized friction factor comparison between the original and optimal cases.
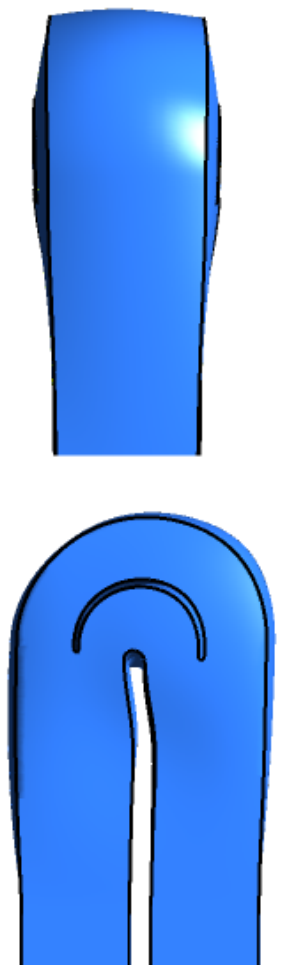

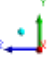

$\stackrel{\leftrightarrow}{\longrightarrow}$

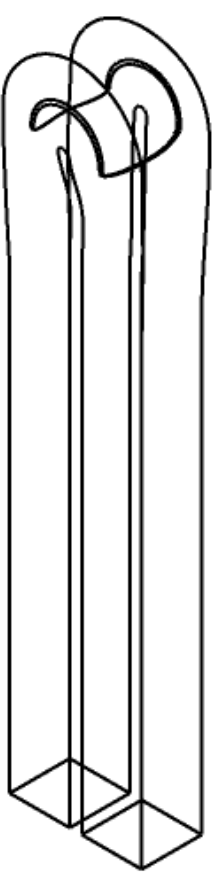

Fig. 7. Optimal GV-1. 


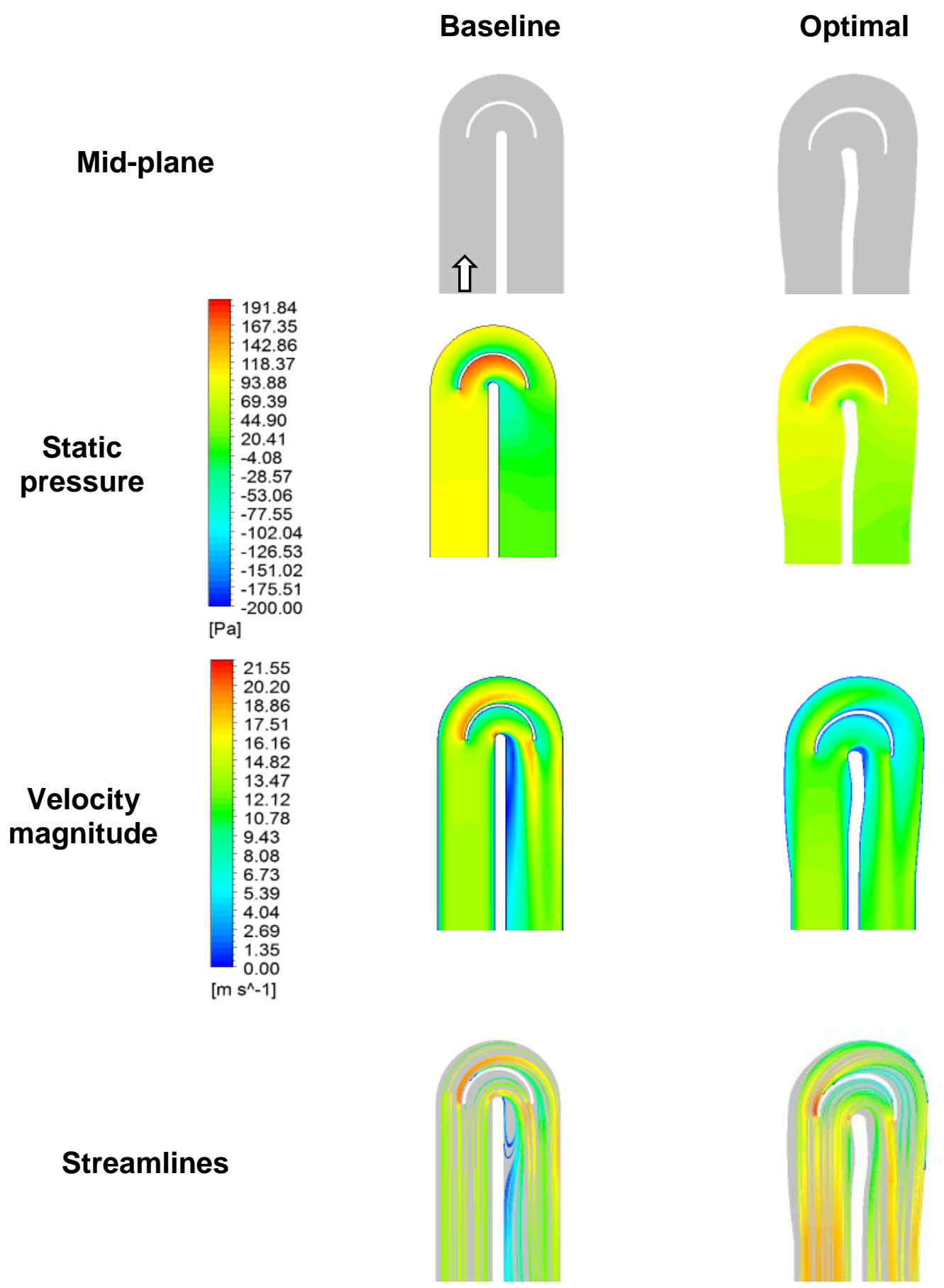

Fig. 8. GV-1 original and the optimal shape at the mid-plane. 

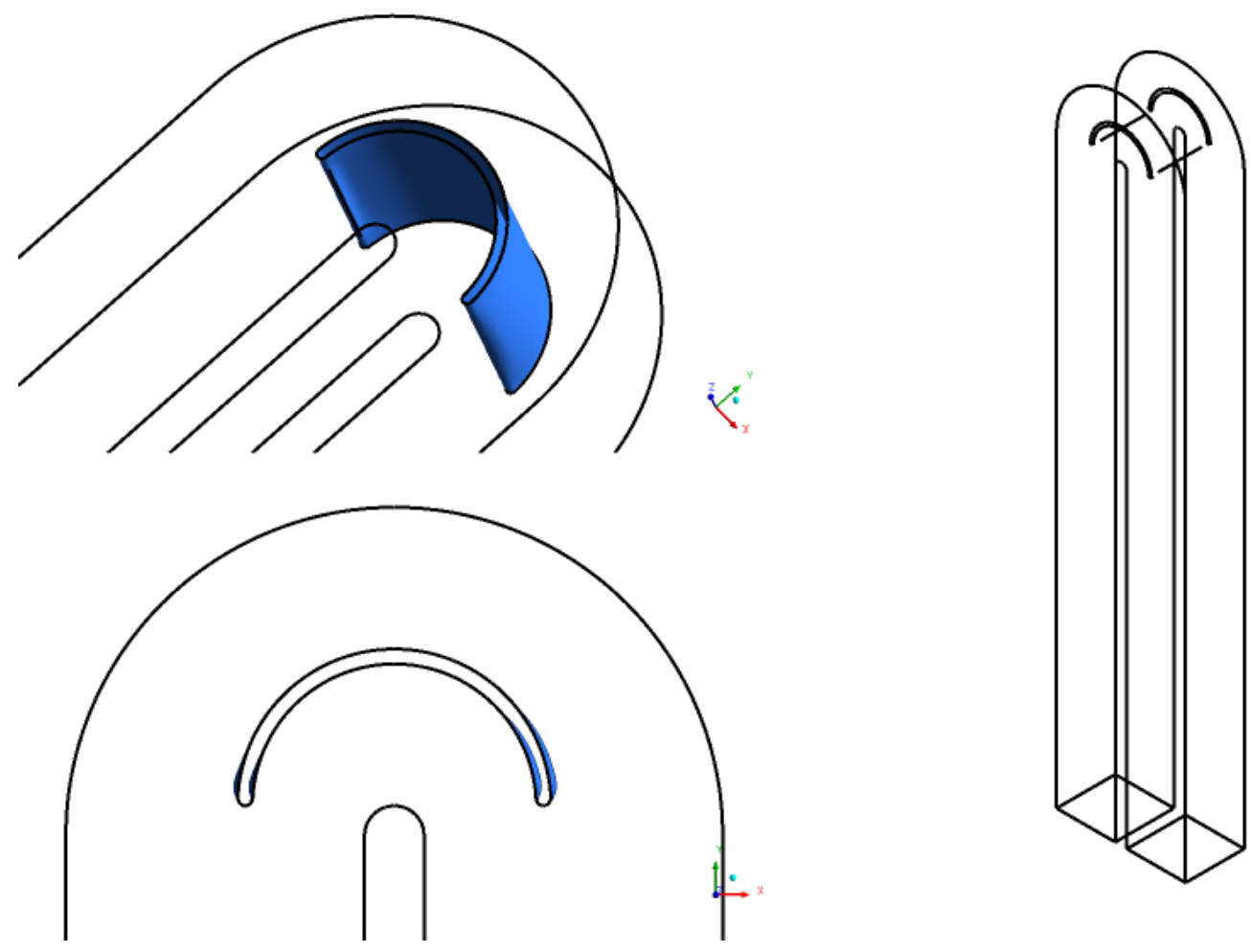

Fig. 9. Optimal GV-2.

Table 3: Comparison between the normalized friction factor for the original and the optimal geometry for the three cases

\begin{tabular}{|l|c|c|}
\hline \multicolumn{1}{|c|}{ Case } & Original $\mathrm{f} / \mathrm{f}_{0}$ & Optimal $\mathrm{f} / \mathrm{f}_{0}$ \\
\hline Without GV & 4.56 & 1.85 \\
\hline GV-1 & 2.6 & 1.75 \\
\hline GV-2 & 2.7 & 2.63 \\
\hline
\end{tabular}

\section{Conclusions}

The adjoint method was used to reduce the pressure drop for three different configurations of U-bend shaped by modifying the shape of these bends according to the part which is allowed to modify and the region to modify. The three geometries after optimization showed enhancement with respect to pressure loss. The best case is guide vane case 1 which produces a pressure loss of $82.14 \mathrm{~Pa}$. On the other hand, the guide vane case 2 represented a limited enhancement by $123.07 \mathrm{~Pa}$ only. The optimal shapes could be manufactured by casting or 3D printer technology. 


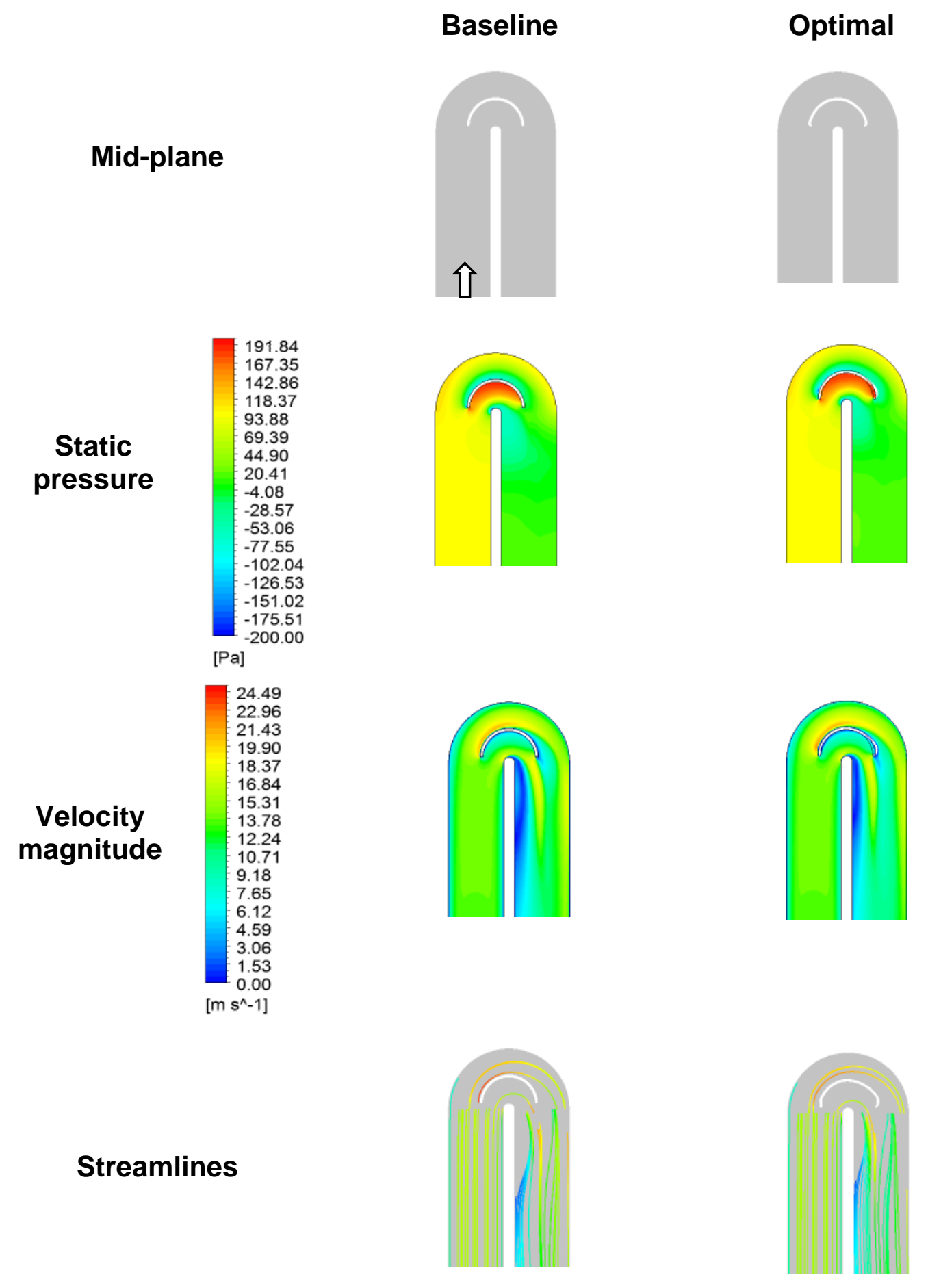

Fig. 10. GV-2 original and the optimal shape at the mid-plane. 

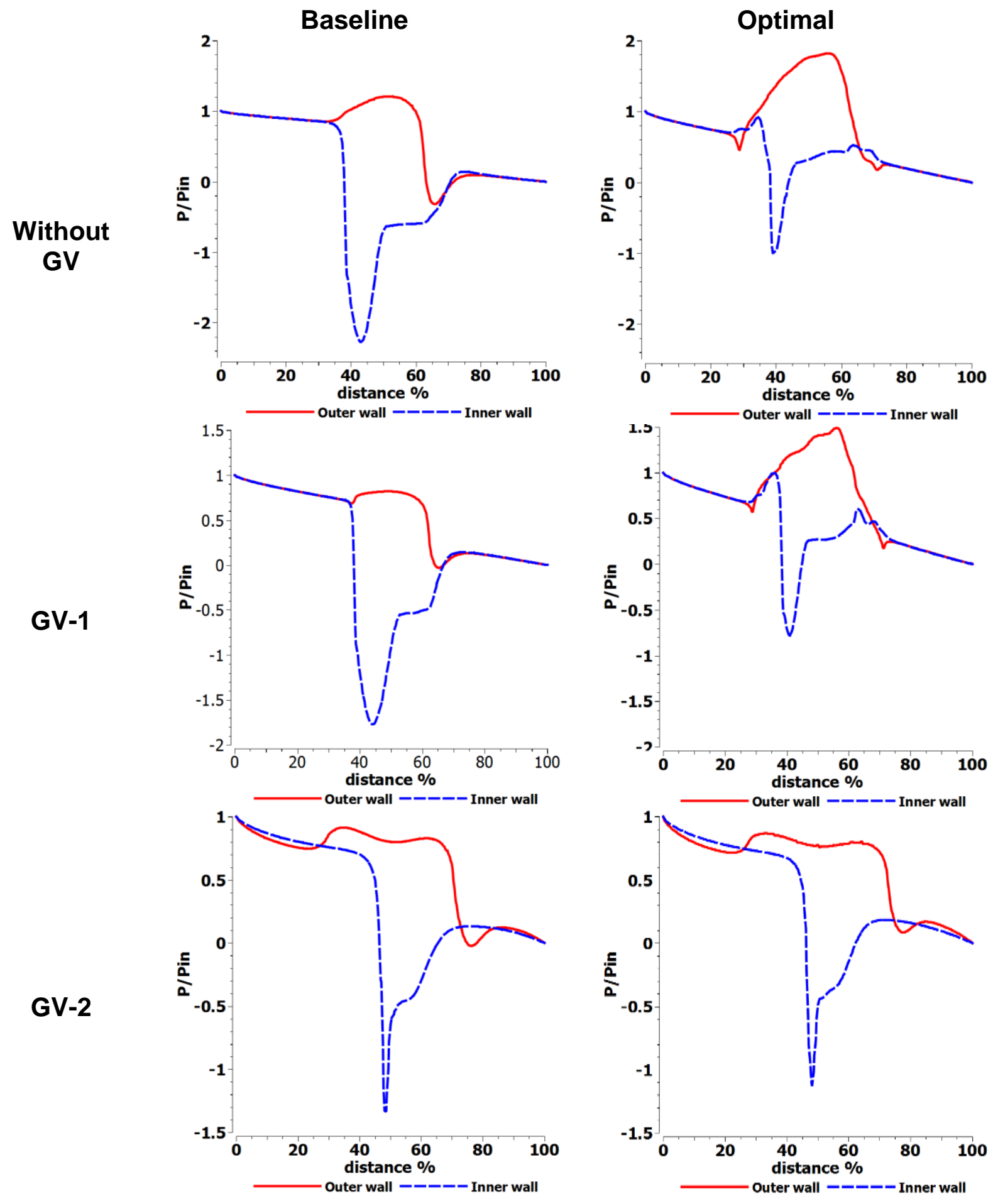

Fig. 11. Pressure distribution along the outer and the inner wall at the mid-plane. 

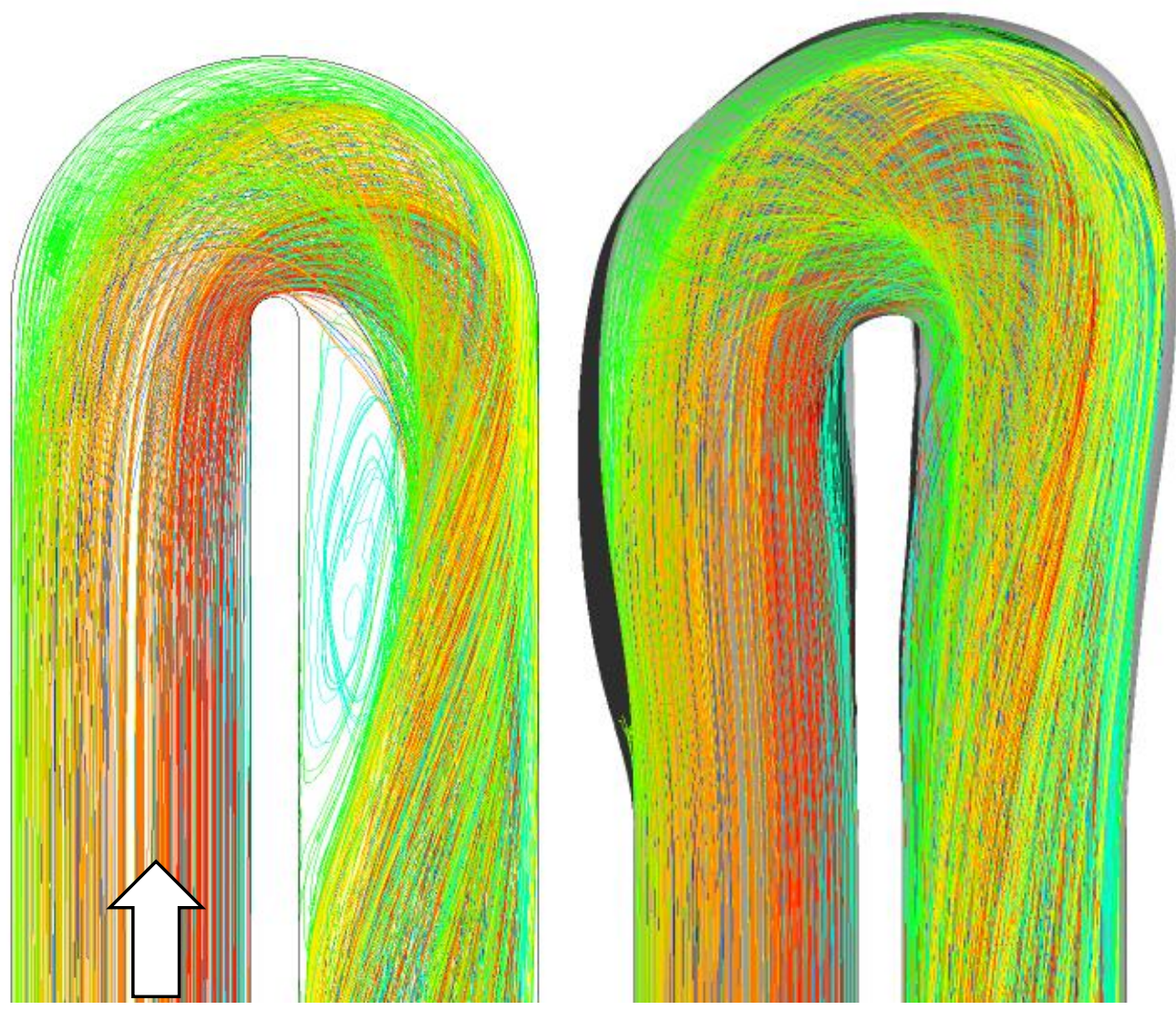

Fig. 12. Pathlines for the original (left) and the optimal shape (right) for case 1.
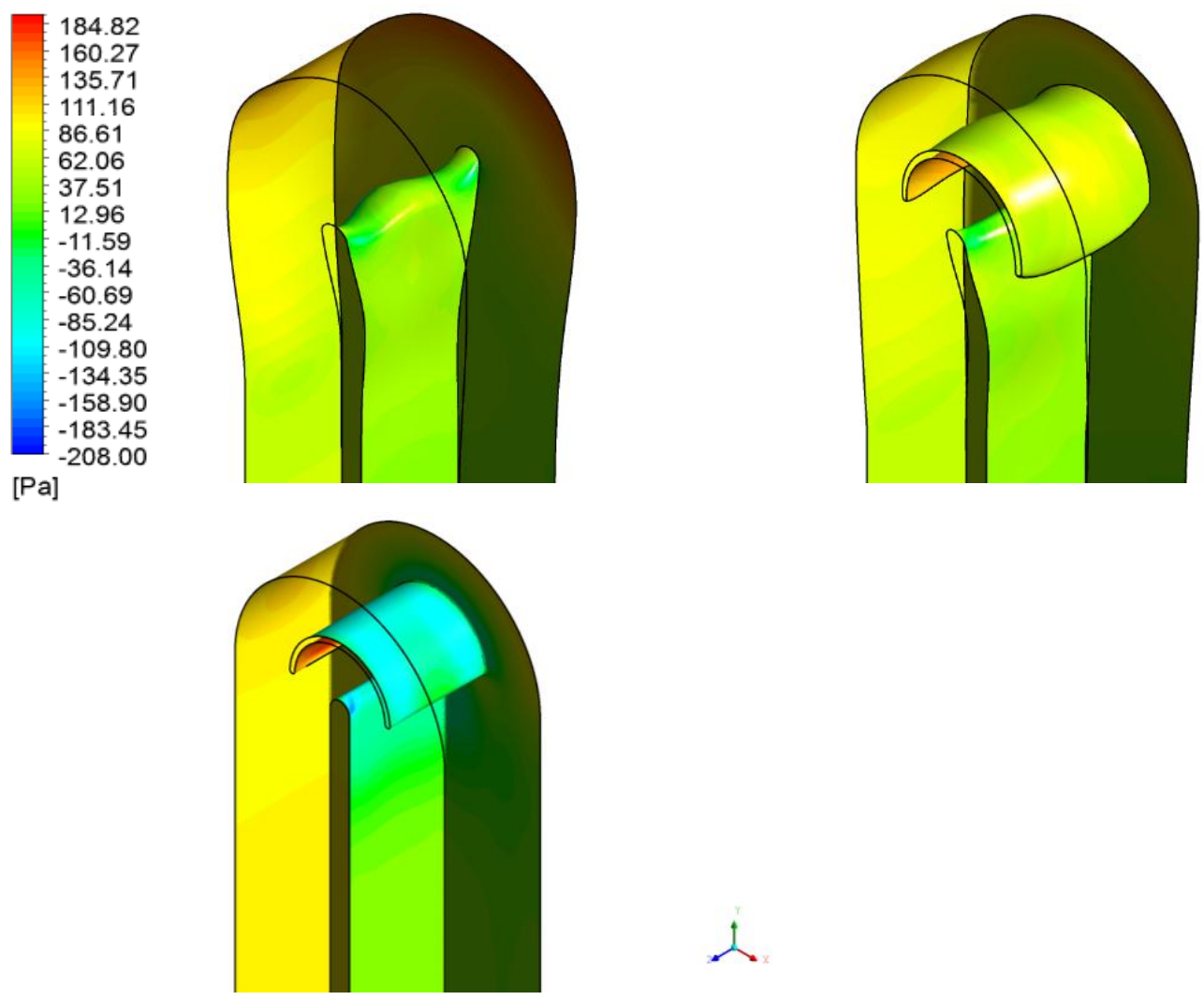

Fig. 13. Pressure contours for the optimal shapes. 


\section{References}

[1] Sudo, K., Sumida, M., and Hibara, H. "Experimental investigation on turbulent flow in a circular-sectioned 180-degree bend," ", Experiments in Fluids, " 2000, pp. 51-57.

[2] Kim, S. and Lee, S.J. "Measurement of Dean flow in a curved micro-tube using micro digital holographic particle tracking velocimetry," ", Experiments in Fluids, " 2009, pp. 255-264.

[3] Miloud, A., Aounallah, M., Belkadi, M., Adjlout, L., Imine, O., and Imine, B. "Turbulent flow computation in a circular U-Bend," 2014, pp. 1-6.

[4] Al-yaari, M.A. and Abu-sharkh, B.F. "CFD Prediction of Oil-Water Phase Separation in 180 o Bend," ", Asian Transactions on Engimeering," 2011, pp. 63-67.

[5] Sewall, E. a and Tafti, D.K. "Large Eddy Simulation of Flow and Heat Transfer in the Developing Flow Region of a Rotating Gas Turbine Blade Internal Cooling Duct With Coriolis and Buoyancy Forces," ", ASME Conference Proceedings, " 2008, pp. 763771.

[6] Luo, J. and Razinsky, E.H. "Analysis of Turbulent Flow in 180 deg Turning Ducts With and Without Guide Vanes," ", ASME Conference Proceedings, " 2007, pp. 899908.

[7] Schüler, M., Zehnder, F., Weigand, B., von Wolfersdorf, J., and Neumann, S.O. "The Effect of Turning Vanes on Pressure Loss and Heat Transfer of a Ribbed Rectangular Two-Pass Internal Cooling Channel,", Journal of Turbomachinery, " 2011, pp. 21017.

[8] Saha, K. and Acharya, S. "Effect of Bend Geometry on Heat Transfer and Pressure Drop in a Two-Pass Coolant Square Channel for a Turbine," ", Journal of Turbomachinery," 2013, pp. 1-12.

[9] Lee, D.M., Park, J.S., Lee, D.H., Lee, S., Soo Kim, B., and Cho, H.H. "Heat-Transfer Characteristics of a Non-Rotating Two-Pass Rectangular Duct With Various Guide Vanes in the Tip Turn Region," ", Journal of Turbomachinery," 2012, pp. 51039.

[10] Xie, G., Zhang, W., and Sunden, B. "Computational analysis of the influences of guide ribs/vanes on enhanced heat transfer of a turbine blade tip-wall," ", International Journal of Thermal Sciences, " 2012, pp. 184-194.

[11] Verstraete, T. and Li, J. "Multi-Objective Optimization of a U-bend For Minimal Pressure loss and Maximal Heat Transfer Performance in Internal Cooling Channels," ", ASME Turbo Expo 2013 Power Land, Sea Air," San Antonio, Texas, USA,pp. 1-12.

[12] Elsayed, K. and Joao Miranda, C.L. " Minimization of The pressure Loss In Internal Cooling Channels Using The Adloint Method," ", 1st Aviation Engineering Innovations Conference 2015," Cairo, EGYPT,pp. 1-9. 